\title{
Construcción sintáctica en el encabezamiento de las historias clínicas de Epidemias II, IV y VI ${ }^{1}$
}

\author{
Alicia Esteban Santos \\ Universidad Complutense de Madrid \\ aesteban@filol.ucm.es
}

Recibido: 14-11-2011

Aceptado: 30-11-2011

\section{RESUMEN}

En este trabajo examinamos las distintas construcciones sintácticas utilizadas en el encabezamiento de las historias clínicas de Epidemias II, IV y VI para referirse al paciente y determinar su identidad: el caso en que se le menciona, el uso del artículo en su designación, el empleo del participio y de la oración de relativo, que son a veces el único medio de referencia al paciente, y, en fin, por otra parte, los diversos giros sintácticos, más o menos complejos, que se utilizan en su designación. Los resultados en todas estas cuestiones estudiadas apuntan en la misma dirección: una conexión mucho mayor entre los libros II y VI de Epidemias, frente a Epid. IV, que se muestra en notable divergencia.

Palabras clave: Epidemias hipocráticas: libros II, IV y VI; historias clínicas, esquema de composición, divergencias y paralelismos, sintaxis griega.

\begin{abstract}
In this essay we examine the different syntactical constructions used in the beginning of the clinical histories of Epidemics II, IV and VI to refer to the patient and to determine his identity: the case in which he is mentioned, the use of article in his designation, the employment of participle and relative, that are sometimes the only way of reference to the patient, and, on the other hand, the diverse syntactical constructions, more or less complex, which are used in his designation. The results in all these questions point in the same direction: a stronger connection between the books II and VI of Epidemics, in contrast to Epid. IV, which is shown in remarkable divergence.
\end{abstract}

Key words: Hippocratic Epidemics: Books II, IV and VI; clinical histories, scheme of composition, divergences and parallels, Greek Syntax.

Intentamos ir precisando así - paso a paso - el esquema de composición de las historias clínicas de Epidemias II, IV y VI, según el método establecido para nuestro estudio de las historias clínicas de Epidemias I y III, por un lado, y de Epidemias V y VII,

\footnotetext{
${ }^{1}$ Este trabajo pertenece al proyecto DGICYT (FFI2009-10118) «Estudios sobre el Corpus Hippocraticum y su influencia», bajo la dirección del Dr. Ignacio Rodríguez Alfageme.
} 
por otro ${ }^{2}$. Comenzamos con los datos del encabezamiento, y más concretamente con la manera de identificar a los pacientes de este grupo de tratados, Epidemias II, IV y VI, porque la alusión al paciente casi siempre inicia las historias clínicas, tanto en este grupo como en los otros dos, y del examen de esta cuestión se pueden obtener interesantes resultados, principalmente para determinar las divergencias y paralelismos entre los distintos tratados.

Como ya precisamos con respecto a Epid. $\mathrm{V} \mathrm{y} \mathrm{VII}^{3}$, son varios los aspectos a tomar en consideración. De éstos nos parecen fundamentalmente relevantes:

1) La designación en sí del paciente, ya identificándole con exactitud mediante un nombre propio (el suyo o el de un allegado: padre, esposo, hermano, hijo, amo), ya de manera más o menos anónima e indeterminada.

2) La referencia al paciente con respecto a otro personaje, en una relación de parentesco o de servidumbre. Es lo más frecuente en el caso de mujeres: «mujer de», «hija de», «hermana de», «pariente de». También con respecto a hijos y servidores ${ }^{4}$.

3) Usos sintácticos diversos:

- El caso gramatical en el que se halla la designación del paciente, por lo general en nominativo o dativo (y mucho más raramente en genitivo o acusativo).

- El uso del artículo que determina al paciente.

- Construcciones empleadas.

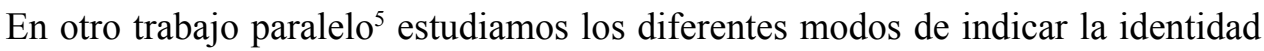
del paciente en Epid. II, IV y VI concernientes a aspectos más bien de contenido: el empleo del nombre propio (el suyo o el de un allegado), o bien la designación más o menos indeterminada; las relaciones de parentesco o servidumbre; las características (edad, profesión, domicilio, etc.) y circunstancias diversas — en ocasiones algo anecdóticas incluso - que se indican sobre el paciente, así como el lugar y época en que se produce su afección. Todos estos datos son independientes del desarrollo de la enfermedad, y muestran la visión que da el autor — el médico — de la personalidad del paciente, sobre todo para poder identificarlo.

${ }^{2}$ Pues en las historias clínicas (en Epidemias I y III especialmente) observamos un tipo de composición común, con datos fijos que se presentan en general en el mismo orden y que con frecuencia son expresados mediante frases hechas o fórmulas. Es lo que llamo «esquema fijo». Son los principales datos que configuran el tal esquema unos «externos» a la enfermedad en sí, en indicación del paciente, la ciudad y la época del año, y otros ya se refieren a la propia enfermedad y su desarrollo: causa, indicación inicial de la enfermedad, descripción de los diversos síntomas (destacando fiebre, dolor y trastornos psíquicos), altibajos, terapia, el transcurrir del tiempo, desenlace, reflexiones post desenlace. Cf. Esteban Santos (1994: 50-69 y 1998, entre otros).

${ }^{3}$ Cf. Esteban Santos 1998: 416ss.

${ }^{4}$ Jouanna (1992: 163) señala que de los más de 450 casos individuales recogidos en las Epidemias muchos son individualizados de manera que se puede determinar a menudo su origen social: cuando se da el nombre propio es porque se trata de un hombre libre, ya sea él mismo el enfermo o un miembro de su familia (mujer, hijo, etc.), y otros muchos son designados expresamente como «esclavo de», «servidor de».

${ }^{5}$ Esteban Santos (en prensa). 
En el presente estudio completamos el examen centrándonos principalmente en cuestiones de forma, y dedicamos nuestra atención ante todo a los distintos usos sintácticos y construcciones utilizadas en el encabezamiento en alusión al paciente.

Como también en los trabajos anteriores sobre Epidemias II, IV y VI, tomamos en consideración a los pacientes de todos los pasajes referentes a enfermos individuales: no sólo de las historias clínicas propiamente dichas, sino también de los pasajes de mera mención de pacientes como ejemplo o comparación ${ }^{6}$, pues entre unos y otros no existe diferencia sustancial en cuanto a la manera de citar al paciente. Y asimismo como en los trabajos anteriores es objetivo importante en éste mostrar las discrepancias y puntos en común entre los distintos libros. Así pues, tras examinar las diversas cuestiones tratado por tratado, recapitularemos sobre las divergencias que se observan entre ellos:

\section{- En Epidemias II:}

El número total de enfermos mencionados es 41 (contabilizamos de nuevo al mismo enfermo si aparece citado en otro pasaje ${ }^{7}$, pero no cuando se repite su mención en el mismo contexto), de los que 22 son hombres y 19 mujeres.

Caso gramatical en que aparece la designación del paciente: en Nominativo, 18 (19 en Smith) ${ }^{8}$; en Dativo, 17 (15 Smith); en Genitivo, 6 (7 Smith), de los que 5

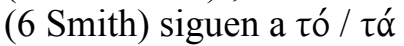

Artículo: 15 enfermos en total aparecen con artículo: en 10 casos éste acompaña al nombre de designación del paciente (de los que en 9 se trata del nombre común y en 1, probablemente, del nombre propio o gentilicio ${ }^{9}$ ); pero en 5 casos el paciente es designado sin nombre, únicamente mediante el artículo referido a él, que en 4 casos antecede al nombre en Genitivo posesivo de un allegado ( 3 nombre propio y 1 nombre común), y en 1 caso acompaña a un participio.

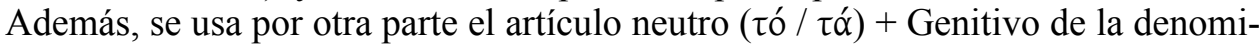
nación del paciente en 5 (6 en Smith) casos.

Participios en referencia al paciente: 3 sin artículo (sin otra indicación de referencia al paciente), y 1 con artículo

Relativos en referencia al paciente: 1 sin otra designación. Además, otros 2 (1 Smith) añadidos a otra designación.

En algunos casos la construcción puede ser algo más compleja, sumando más de un procedimiento de identificación del paciente, como después examinaremos con más detenimiento.

${ }^{6}$ Cf. Esteban Santos (2011: 182ss.). Nikitas (1968: 52ss.) trata de los casos o historias clínicas de Epid. II, IV y VI, y distingue los enfermos como ejemplo (oĩov), estableciendo en esta categoría de pasajes tres diferentes tipos.

${ }^{7}$ Así, en los textos paralelos. Sobre éstos, $c f$. Nikitas (1968: 125ss.)

${ }^{8}$ Según la edición de Smith (1994), frente a la de Langholf (1977), que seguimos en general para las historias clínicas de Epid. II y IV. Pero en la edición de Langholf no se incluyen los pasajes de mera mención de pacientes como ejemplo o comparación.

${ }_{9}^{9}$ Aunque no queda claro, porque el texto está corrupto: Epid. II 2,3: Littré 5 84,8 = Langholf 1977, 114, párrafo $3=$ Smith 30,15 . 
- En Epidemias IV:

El número total de enfermos mencionados es $111^{10}$, de los que 72 son hombres y 39 mujeres

Caso gramatical: en Nominativo, 63; en Dativo, 40; en Genitivo, 5, de los que 1 sigue a tó; en Acusativo, 1.

Con Artículo: 77 enfermos en total aparecen con artículo: en 44 casos éste acompaña al nombre de designación del paciente (de los que en 37 se trata del nombre común y en 7 , del nombre propio o gentilicio ${ }^{11}$ ); pero en 39 casos el paciente es designado sin nombre, únicamente mediante el artículo referido a él, que en 31 casos antecede al nombre en Genitivo posesivo de un allegado (en 20 casos) o a un giro preposicional (en 11), y en 8 casos acompaña a un participio. Además, se usa por otra parte el artículo neutro (tó) + Genitivo de la denominación del paciente en 1 caso.

Participios con artículo en referencia al paciente: 9. Pero no hay ningún caso de paciente sin otra referencia a él que mediante Participio sin artículo.

Relativos en referencia al paciente: 6 sin otra designación. Además, otros 14 añadidos a otra designación.

En algunos casos la construcción puede ser algo más compleja, sumando más de un procedimiento de identificación del paciente, como después examinaremos con más detenimiento.

\section{- En Epidemias VI:}

El número total de enfermos mencionados es 36 ( 35 en $\left.\mathrm{Smith}^{12}\right)$, de los que 28 son hombres y 8 mujeres (7 Smith)

Caso gramatical: en Nominativo, 15; en Dativo, 18 (17 Smith); en Genitivo, 2, que siguen a tó; en Acusativo, 1.

Con Artículo: 13 enfermos en total aparecen con artículo: en 9 casos éste acompaña al nombre de designación del paciente (de los que en 5 se trata del nombre común y en 4 , del nombre propio o gentilicio); pero en 4 casos el paciente es designado sin nombre, únicamente mediante el artículo referido a él, que en 3 casos antecede al nombre en Genitivo posesivo de un allegado (en 1 caso) o a un giro preposicional (en 2), y en 1 caso acompaña a un participio.

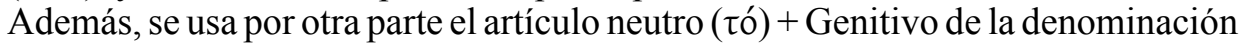
del paciente en 2 casos.

${ }^{10}$ Pero no es fácil determinar el número exacto. Según Robert (1975: 180) hay 117 casos individuales en Epid. IV. Quizás porque contabiliza más de una vez los casos en que se menciona reiteradamente a un paciente en el mismo contexto, lo que no hacemos en este trabajo, como ya indicamos.

${ }^{11}$ Englobamos en una misma estas dos categorías diferentes porque en ocasiones es difícil saber si se trata de una u otra.

${ }^{12}$ Según la edición de Smith (1994), frente a la de Manetti \& Roselli (1982), que seguimos en general para las historias clínicas de Epid. VI. 
Participios en referencia al paciente: 2 sin artículo $^{13}$ (sin otra indicación de referencia al paciente), y 1 con artículo.

Relativos en referencia al paciente: 6 (5 Smith) sin otra designación, y 2 añadidos al sustantivo indeterminado «hombre»/«mujer» con artículo, lo que viene a ser equivalente.

En algunos casos la construcción puede ser algo más compleja, sumando más de un procedimiento de identificación del paciente, como después examinaremos con más detenimiento.

Ahora comprobemos los resultados de cada uno de los aspectos examinados para establecer las diferencias específicas entre los tratados del grupo de Epidemias II, IV y VI:

\section{Caso gramatical}

- En Epidemias II (número total 41 pacientes):

Pacientes designados en Nominativo, 18 (19 Smith) [43,9\%] ${ }^{14}$; en Dativo, 17 (15 Smith) [41,46\%]; en Genitivo, 6 (7 Smith), de los que 5 (6 Smith) siguen a tó / $\tau$ ó. Ninguno en Acusativo.

- En Epidemias IV (número total 111 pacientes):

Pacientes designados en Nominativo, 63 [56,3\%]; en Dativo, 38 [34,2\%]; en Genitivo, 5, de los que 1 sigue a tó; en Acusativo, 1.

- En Epidemias VI (número total 36 (35 Smith) pacientes):

Pacientes designados en Nominativo, 15 [41,66\% $]^{15}$; en Dativo, 18 (17 Smith) [50\%]; en Genitivo, 2, que siguen a тó; en Acusativo, 1.

\section{EN CONCLUSIÓN:}

En Epidemias IV hay notable desproporción entre el Nominativo, 63 [56,3\%] y el Dativo, 38 [34,2\% $]^{16}$; mientras que en Epidemias II (sobre todo) y VI están bastante equilibrados Nominativo y Dativo, aunque predomina un poco el Nominativo en Epidemias II y el Dativo en cambio en Epidemias $\mathrm{VI}^{17}$.

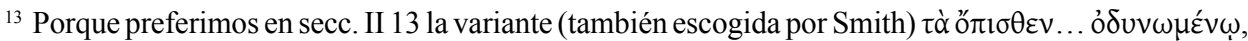
en vez de $\tau \tilde{\omega}$ ŏ $\pi \sigma \theta \varepsilon v . .$. ó $\delta v \nu \omega \mu \varepsilon ́ v \omega$, ya que nos parece más acorde con el estilo de Epid. VI, en donde

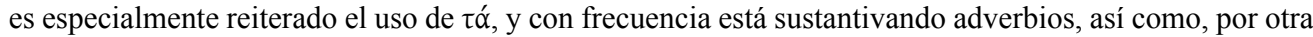
parte, comienza a menudo la frase, como aquí.

${ }^{14}$ Establecemos la proporción de acuerdo con los datos de la edición de Langholf (1977).

${ }^{15}$ Establecemos la proporción de acuerdo con los datos de la edición de MANETTI \& RoselLi (1982).

${ }^{16}$ Sin embargo, Deichgräber $\left(1971^{2}: 65\right)$, que considera que los tres tratados son del mismo autor, comenta respecto a las historias de Epid. II, IV y VI (a propósito de la frecuencia del dativo simpatético en estos tratados en vez del genitivo) que en la mayoría de los casos se comienza con la designación del paciente en dativo, y que el dativo no es tan frecuente sin embargo ni en Epidemias I y III ni en los libros V y VII. Así es en Epid. I y III; pero, en lo referente a Epid. V y VII, mientras que el dativo es muy poco usado en efecto en las historias exclusivas del libro $\mathrm{V}$ para aludir al paciente, en las historias exclusivas del libro VII y en las «historias paralelas» es, por el contrario, el más utilizado. Cf. Esteban Santos 1998: 417.

17 También en Epid. V y VII la desproporción en el uso de Nominativo o Dativo en la designación del paciente es un dato significativo para establecer las diferencias entre los tres grupos de textos (los exclusivos del libro V, los exclusivos del libro VII y los «textos paralelos»), y se observa en esta cuestión 


\section{Uso del artículo}

- En Epidemias II (número total 41 pacientes):

15 enfermos [36,58\%] aparecen con artículo: en 10 casos acompaña al nombre de designación del paciente [24,39\%]; en 5 casos no acompaña a su nombre, sino a otra construcción [12,19\%].

- En Epidemias IV (número total 111 pacientes):

77 enfermos [69,8\%] aparecen con artículo: en 44 casos acompaña al nombre de designación del paciente [39,63\%]; en 39 casos no acompaña a su nombre, sino a otra construcción $[35,1 \%]$.

- En Epidemias VI (número total 36 (35 Smith) pacientes):

13 enfermos [36,1\%] aparecen con artículo: en 9 casos acompaña al nombre de designación del paciente [25\%]; en 4 casos no acompaña a su nombre, sino a otra construcción $[11,11 \%]$.

\section{EN CONCLUSIÓN:}

En Epidemias II y VI —en casi exacta proporción entre sí- sólo en una tercera parte aproximadamente aparecen los enfermos determinados por el artículo, frente a Epidemias IV, en que sucede en más de las dos terceras partes (aunque eso está en consonancia con el hecho de que en el libro IV son menos los enfermos designados mediante su nombre propio ${ }^{18}$, que no suelen llevar artículo tan a menudo). Además, en Epidemias IV es mucho mayor la proporción que en Epidemias II y VI de casos en que el artículo que determina al paciente no acompaña al nombre común o propio que lo designa, sino a otro tipo de construcción (giro, participio), pues están más o menos equilibradas en uno u otro caso, mientras que en Epidemias II y VI el artículo con el nombre aparece en doble proporción que con otro tipo de construcción ${ }^{19}$.

\section{Uso del participio}

- En Epidemias II (número total 41 pacientes):

Participios sin artículo (sin otra indicación de referencia al paciente): 3; Participios con artículo: 1.

- En Epidemias IV (número total 111 pacientes):

Participio sin artículo: ninguno; Participios con artículo: 9.

- En Epidemias VI (número total 36 (35 Smith) pacientes):

una mayor coincidencia entre los textos paralelos y los exclusivos de Epid. VII. Cf. Esteban Santos (1998: 417). Eso está en conformidad con los resultados obtenidos en nuestros otros estudios sobre Epid. V y VII. $C f$. asimismo los trabajos de Ángel Espinós (2002, 2003 y 2009).

${ }^{18} C f$. Esteban Santos (en prensa).

${ }^{19}$ También en Epid. V y VII la desproporción en el uso del artículo en la designación del paciente es un dato significativo para establecer las diferencias entre los tres grupos de textos, y se observa asimismo en esta cuestión una mayor coincidencia entre los textos paralelos y los exclusivos de Epid. VII, frente a los exclusivos del libro V. $C f$. Esteban Santos 1998: 417s. Eso está en conformidad con los resultados obtenidos en nuestros otros estudios sobre Epid. V y VII. 
Participios sin artículo (sin otra indicación de referencia al paciente): 2; Participios con artículo: 1 .

\section{EN CONCLUSIÓN:}

La diferencia es sobresaliente, y radica, por un lado, en el hecho de que tanto en Epidemias II como VI aparecen algunos casos en que el paciente es identificado únicamente mediante un participio sin artículo (y en mayor proporción que mediante participio con artículo), mientras que entre las tan numerosas historias de Epidemias IV no se da en ningún caso. Por otra parte, sin embargo, es bastante mayor la proporción de casos en que aparece participio con artículo (en referencia al paciente en el encabezamiento) en Epidemias IV [8,1\%] que en Epidemias II y VI [2,43\% y 2,77\% respectivamente]

\section{Uso del relativo}

- En Epidemias II (número total 41 pacientes):

Relativos: 1 sin otra designación [2,43\%]. Además, otros 2 (1 Smith) añadidos a otra designación $[4,87 \%]$.

- En Epidemias IV (número total 111 pacientes):

Relativos: 6 sin otra designación [5,4\%]. Además, otros 14 añadidos a otra designación [12,61\%] (en uno de los casos, además, se agrega nuevo relativo, en construcción más compleja [en cap. 38]).

- En Epidemias VI (número total 36 (35 Smith) pacientes):

Relativos: 6 (5 Smith) sin otra designación [16,66\%], y 2 añadidos a otra designación $[5,55 \%]$, aunque ésta es el sustantivo indeterminado-«hombre»/ «mujer» con artículo.

EN CONCLUSIÓN:

El uso del relativo en general para designar al paciente se muestra menos frecuente en Epidemias II, que en este aspecto difiere de Epidemias VI aún más que de Epidemias IV. Pero de nuevo Epidemias II y VI se aproximan entre sí frente a Epidemias IV en el hecho de que son pocos los casos en se añade el relativo a otra designación, mientras que abundan en Epidemias IV (aprox. 2'5 veces más en proporción).

\section{Giros sintácticos y construcciones complejas}

En lo que respecta a las diversas construcciones sintácticas empleadas para indicar la identidad del paciente, podemos observar distintas preferencias de uso y mayor o menor complejidad en los diferentes tratados:

- En Epidemias II (número total 41 pacientes):

— En 7 casos [17,07\%] se utiliza la construcción: art. paciente + gen. allegado

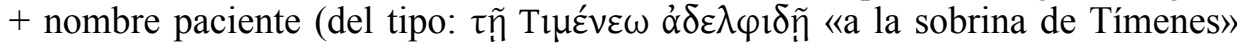
[secc. I 7] ${ }^{20}$ ). En 3 casos es así simplemente, y en otros 4 con alguna complejidad,

\footnotetext{
${ }^{20}$ Epid. II 1,7: Littré 5 78,9 = Smith 24,14.
} 
ampliando los datos del allegado [secc. II 6 y secc. II 9] o los del paciente, en construcción más compleja [secc. III 4 y secc. IV 5, que después examinaremos]

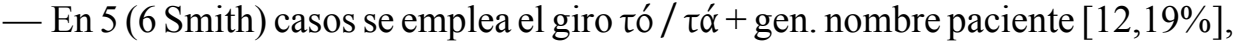

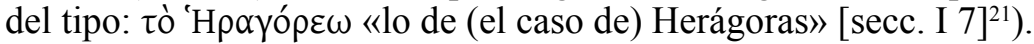

- Por lo demás, las construcciones en su mayoría son simples (con un solo procedimiento de identificación del paciente), excepto en 5 (4 Smith) casos [12,19\%] más complejos (de los que acabamos de mencionar algunos), en que se utiliza más de un procedimiento de identificación del paciente. Así, añadiendo una nueva precisión sobre él precedida de artículo: art. paciente + gen. allegado $1 .^{\circ} /+$ art. paciente + giro con nombre allegado $2 .^{\circ}$ (repitiendo el artículo, sin

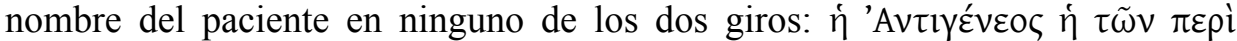

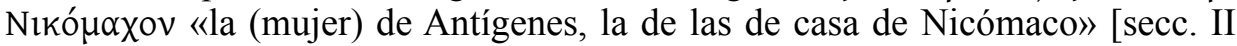
$19{ }^{22}$ ); o bien art. paciente + gen. allegado / + art. paciente + nombre paciente

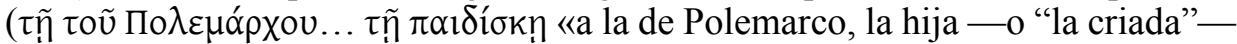
jovencita» [secc. III 4] ${ }^{23}$ ); o bien nombre propio paciente sin artículo / + art.

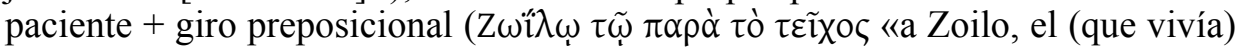
junto a la muralla» [secc. III 3$]^{24}$ ). O es introducida una nueva precisión mediante

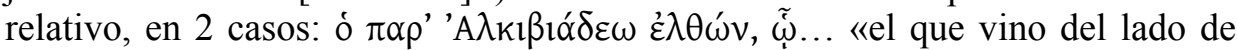
Alcibíades, al que...» (art. paciente + giro preposicional + Partic. / + Relat.)

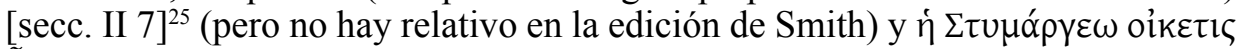
$\tilde{\eta}$ «la criada de Estimarges, a la que...» (art. paciente + gen. allegado + nombre paciente / + Relat.) [secc. IV 5] $]^{26}$.

- En Epidemias IV (número total 111 pacientes):

— En 10 casos [9\%] se usa la construcción: art. paciente + gen. allegado + nombre paciente. Pero en uno de éstos aparece ampliado dentro de una construcción más compleja [cap. 25] que después examinaremos. La construcción algo semejante: art. paciente + giro prepos. + nombre paciente aparece en 8 casos [7,2\%], como por ejemplo: $\tau \tilde{n}$ ő Heroon» [cap. 23] ${ }^{27}$. Uno de ellos está ampliado dentro de una construcción más compleja [cap. 40] que después examinaremos. De este tipo exacto no aparece ninguno ni en Epidemias II ni en Epidemias VI.

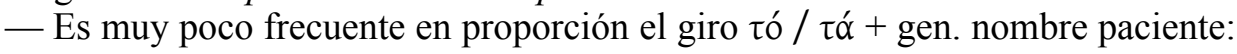
en sólo 2 casos $[1,8 \%]$.

- En 24 casos en total [21,6\%] las construcciones son complejas, porque se utiliza más de un procedimiento de identificación del paciente. Así, añadiendo una nueva precisión sobre él repitiendo el artículo: art. paciente + nombre paciente

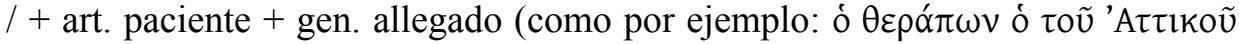

${ }^{21}$ Epid. II 1,7: Littré 5 78,18 = Smith 26,5.

${ }^{22}$ Epid. II 2,19: Littré 5 92,3 = Langholf 1977, 120, párrafo $27=$ Smith 36,16.

${ }^{23}$ Epid. II 3,4: Littré 5 108,5-6 = Smith 52,16-7.

${ }^{24}$ Epid. II 3,3: Littré 5 104,13 = Langholf 1977, 124, párrafo $41=$ Smith 50,9.

${ }^{25}$ Epid. II 2,7: Littré 5 86,15 = Langholf 1977, 116, párrafo $14=$ Smith 32,15.

${ }^{26}$ Epid. II 4,5: Littré 5 126,10 = Langholf 1977, 131, párrafo $63=$ Smith 72,8.

${ }^{27}$ Epid. IV 23: Littré 5 162,16 = Langholf 1977, 149, párrafo $162=$ Smith 114,16. 
«el criado, el del Ático» [cap. 13] ${ }^{28}$ ), en 5 casos, aunque en 1 de ellos [en cap. 21] está ampliado, como veremos a continuación; y en otro caso se invierte el orden (art. paciente + gen. allegado $/+$ art. paciente + nombre paciente [en cap. 31]), y está además aún ampliado, como veremos a continuación; y en otro caso se añade nuevo sustantivo (art. paciente + nombre paciente $/+$ art. paciente + gen. allegado + nombre paciente [en cap. 25]), y está además aún ampliado, como veremos a continuación. $\mathrm{O}$ bien, asimismo repitiendo el artículo: art. paciente + nombre paciente / + art. paciente + giro preposicional, en 2 casos [en cap. 20 y cap. 30]; o art. paciente + giro preposicional + nombre paciente $/+$ art. paciente + gen. allegado [en cap. 40]; o bien art. paciente + nombre paciente $/+$ art. paciente + participio, en 3 casos [en cap. 19, cap. 50 y cap. 55]. Sin repetición del artículo aparecen otras construcciones diversas: nombre propio paciente / + art. paciente + gen. allegado [cap. 4]; o nombre propio paciente / + art. paciente + nombre paciente (indicando profesión) [cap. 23]; o art. paciente + participio / + nombre paciente (indicando edad) [cap. 15], y, principalmente, es introducida una nueva precisión mediante relativo, en 13 casos, de los que algunos (5) muestran una construcción aún más compleja, ampliada con diversas precisiones, como veremos a continuación.

De los 24 casos contabilizados de construcciones complejas, 5 casos [el $20,8 \%$ de las construcciones complejas y el $4,5 \%$ del total de casos] presentan construcciones aún más complejas, con tres o más procedimientos de identificación del paciente: art. paciente + nombre paciente $/+$ art. paciente + gen. allegado $/+$

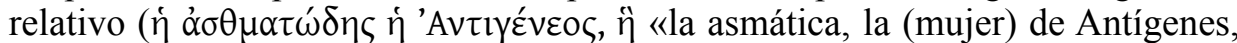

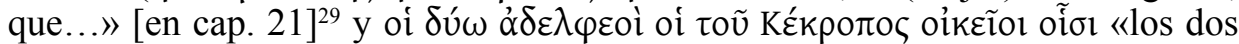
hermanos, los parientes de Cécrope, a los que...» [cap. 25] ${ }^{30}$ ); art. paciente + gen. allegado + nombre paciente $/+$ art. paciente + giro preposicional $/+$ art. paciente

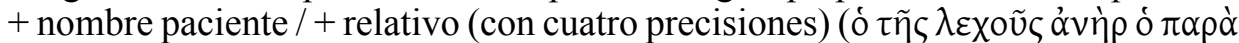

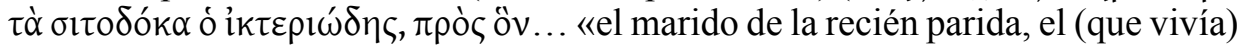
junto a los depósitos de trigo, el afectado de ictericia, al que...» [en cap. 25] ${ }^{31}$ ); art. paciente + gen. allegado $/+$ art. paciente + nombre paciente $/+$ relativo $(\tau \tilde{n}$

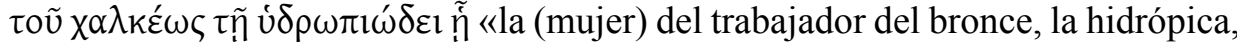
a la que...» [en cap. 31] ${ }^{32}$ ); art. paciente + nombre paciente $/+$ relativo / + relativo

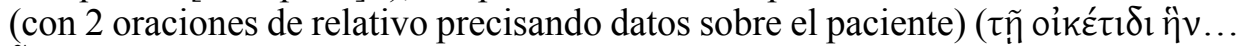
$\tilde{\eta}$... «a la criada que vi..., a la que...» [en cap. 38$]^{33}$ ). Estas construcciones más complejas, con tres o más procedimientos de identificación del paciente, no se dan ni en Epid. II ni en Epid. VI.

- En Epidemias VI (número total 36 (35 Smith) pacientes):

- Es menos frecuente la construcción art. paciente + gen. allegado + nombre paciente, pues aparece en sólo 3 casos [8,33\%], de los que 2 son construcciones

\footnotetext{
${ }^{28}$ Epid. IV 13: Littré 5 150, 19= Langholf 1977, 140, párrafo $104=$ Smith 100, 12-3.

${ }^{29}$ Epid. IV 21: Littré 5 160,14-5 = Langholf 1977, 148, párrafo 153 = Smith 112,14.

${ }^{30}$ Epid. IV 25: Littré 5 164,11 = Langholf 1977, 150, párrafo $168=$ Smith 116,8.

${ }^{31}$ Epid. IV 25: Littré 5 166, 4-5 = Langholf 1977, 150, párrafo 173 = Smith 116,18-9.

${ }^{32}$ Epid. IV 31: Littré 5 174,13 = Langholf 1977, 156, párrafo $216=$ Smith 126,18.

${ }^{33}$ Epid. IV 38: Littré 5 180,5 = Langholf 1977, 160, párrafo 237 = Smith 132,12.
} 
más complejas que se añaden como precisión tras el nombre propio del paciente [ambas en secc. VIII 32, que veremos].

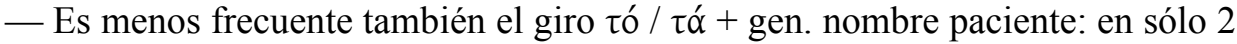
casos $[5,55 \%]$.

- En general las construcciones en su mayoría son simples (con un solo procedimiento de identificación del paciente), como en Epidemias II, excepto en 5 casos [13,8\%] más complejos, en que se utiliza más de un procedimiento de identificación del paciente. Así, añadiendo una nueva precisión sobre el paciente repitiendo el artículo: art. paciente + nombre paciente $/+$ art. paciente + gen.

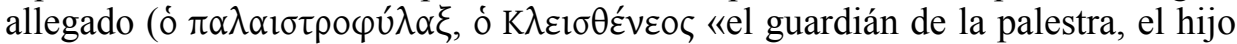
de Clístenes» [secc. VIII 30] ${ }^{34}$; podría interpretarse también con ese esquema

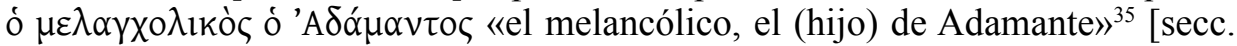
VIII 20] ${ }^{36}$ ); o bien nombre propio paciente sin artículo / + art. paciente + gen.

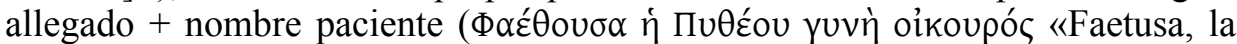

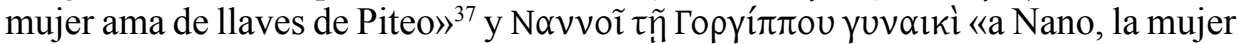
de Gorgico ${ }^{38}{ }^{2}$ [ casos ya mencionados, ambos en secc. VIII 32]). O, en otro

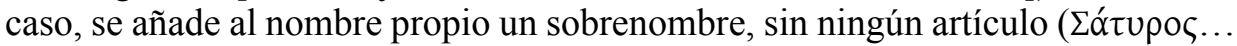
$\gamma \rho \cup \pi \alpha \lambda \omega \tilde{\pi} \eta \xi$ «Sátiro era llamado de sobrenombre Gripalopex» [secc. VIII 29] ${ }^{39}$ ). Respecto a introducir una nueva precisión mediante relativo, en los dos casos en que sucede no se puede considerar propiamente una «nueva precisión» (por ello no los contabilizamos), pues se añade a la designación indeterminada ó ớv $\theta \rho \omega \pi \circ \varsigma$ y

\section{EN CONCLUSIÓN:}

Así pues, aunque difiere Epid. II de Epid. VI en cuanto a la preferencia por las construcciones concretas de «art. paciente + gen. allegado + nombre paciente» y la

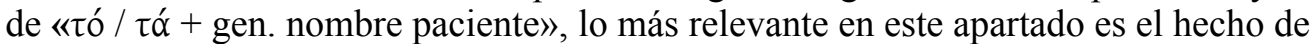
que en Epid. IV es mucho mayor la proporción (casi el doble) que en Epidemias II y VI de casos con una construcción compleja, $\mathrm{y}$, por otra parte, se presentan algunos aún más complejos, con tres o más procedimientos de identificación del paciente, frente a Epidemias II y VI, sin ningún caso en que suceda esto.

${ }^{34}$ Epid. VI 8,30: Littré 5 354,10 = Langholf 1977, 181, párrafo $332=$ Manetti-Roselli 192,4 = Smith 288,4-5.

${ }^{35} \mathrm{Si}$ se considera 'A $\delta \alpha ́ \mu \alpha v \tau o \varsigma$ genitivo (como Manetti-Roselli). O bien sería art. paciente + nombre

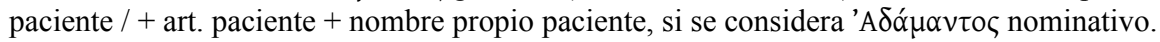
284,19 .

${ }^{36}$ Epid. VI 8,20: Littré 5 352,1 = Langholf 1977, 180, párrafo $328=$ Manetti-Roselli 184,5 = Smith 288,20-21

${ }^{37}$ Epid. VI 8,32: Littré 5 356,4 = Langholf 1977, 182, párrafo 335= Manetti-Roselli 194,5 = Smith 290,6.

${ }^{38}$ Epid. VI 8,32: Littré 5 356,11 = Langholf 1977, 183, párrafo 337 = Manetti-Roselli 194,13 = Smith

${ }^{39}$ Epid. VI 8,29: Littré 5 354,6 = Langholf 1977, 181, párrafo $331=$ Manetti-Roselli 190,5 = Smith 286,21-2. 


\section{CONCLUSIONES FINALES}

Es objetivo importante de este trabajo mostrar las divergencias y puntos de conexión entre los distintos tratados. Se aprecia ante todo la mayor proximidad entre los libros II y VI, frente al otro tratado de este grupo, el IV ${ }^{40}$. A esa conclusión llegamos también en nuestro primer estudio de Epidemias II, IV y VI, acerca de los distintos tipos de pasajes sobre enfermos individuales y su inserción en el todo, al examinar diversos aspectos: la primera diferencia, obvia, es que en el libro IV son los pasajes individuales los predominantes en el texto, mientras que en los libros II y VI abundan más los pasajes generales; pero no radica en la cantidad de casos únicamente, sino en otras numerosas cuestiones además ${ }^{41}$. También en el otro estudio sobre la denominación de los pacientes de Epid. II, IV y VI, atendiendo fundamentalmente a aspectos de contenido, llegamos a resultados análogos: notable contraste entre el libro IV y los libros II y VI, que presentan más puntos en común entre sí.

Ahora hemos examinado las distintas construcciones sintácticas ${ }^{42}$ utilizadas en el encabezamiento de las historias clínicas para referirse al paciente y determinar su iden-

${ }^{40}$ Considerando las relevantes diferencias, es opinión extendida pensar en un distinto autor para el libro IV. Así, Nikitas (1968), Langholf (1977, y en 1990 señala peculiaridades de Epid. IV —cf. p. 222 y, por otra parte, indica - p. 137- que Epidemias II, IV y VI parecen ser en origen notas aisladas de varios autores), Smith (1989 y 1994). García Novo (1989) habla del autor/ autores de Epid. II, IV y VI, pero señala que «el libro IV se distingue de los otros dos tanto por el contenido - fundamentalmente historias - como por el estilo» (p. 11). Sin embargo, Deichgräber $\left(1971^{2}\right)$ advierte en los tres libros - II, IV y VI - una unidad y los cree de un mismo autor (p. 75, y también p. 74). Igualmente Robert (1975), di Benedetto (1977), Jouanna (1989: 65ss.); y, muy recientemente, Alessi (2010) habla siempre en singular del autor de Epidemias II, IV y VI. Por otra parte, Grmek (1980: 217), aunque no considera un autor único, sí un único redactor de todo el conjunto, que supone consistente en un fichero colectivo basado en la experiencia personal de varios médicos. Otros investigadores evitan abordar el tema de la unidad y mencionan Epidemias II, IV y VI como un bloque; así Licciardi (1989): «Sans aborder le sujet de l'identité unique de l'auteur des Epid. II. IV. VI, je me propose...» (p. 117).

${ }^{41}$ En resumen, estos son los resultados a los que llegamos en ese primer estudio respecto al contraste observado entre el libro IV y los libros II y VI: 1. Tanto en el libro II como en el VI es muy elevada la proporción de pasajes individuales de ejemplo o comparación introducidos por oíov o equivalente; pero en el libro IV es mucho menor. 2. Además de la introducción de pasajes individuales mediante oĩov o equivalente en el libro IV (proporcionalmente en menor medida que en los libros II y VI, como acabamos de indicar), se emplea en este tratado una amplia variedad de procedimientos de enlace y referencia en algunas historias clínicas respecto a otros pasajes, en gran contraste con relación a los libros II y VI. 3. En cuanto a la estructura de los pasajes introducidos por oĩov o equivalente, mientras que en los libros II y VI predominan los pasajes en que únicamente se menciona al paciente, sin ningún otro dato, en el libro IV esto ocurre en muy pocos casos, pues en la mayoría se añaden otros datos. 4. Una peculiaridad reiterada en el libro IV no aparece en los libros II y VI: presentar dos historias entremezcladas, con una intercalada en la otra. 5. Mientras que en los libros II y VI predominan con mucho en los pasajes individuales las referencias que indican casos de ejemplificación de la exposición general, en el libro IV señalan en su inmensa mayoría menciones de un paciente en comparación a otro. 6. Por otra parte, cuando los pasajes individuales se hallan como ejemplos de la exposición general, en el libro IV hacen referencia a pasajes generales «de pasado» casi tanto como «de presente», mientras que en los libros II y VI son casi exclusivamente «de presente», aludiendo a hechos de validez universal.

${ }^{42}$ Nikitas (1968: 115ss.) examina las diferencias lingüísticas y estilísticas que distinguen Epidemias IV de II y VI; entre ellas, ciertos usos del artículo. Éstas, unidas a las diferencias de contenido, le llevan a pensar en un distinto autor de Epid. IV ( $c f .234$ ss.). 
tidad: el caso en que se le menciona, el uso o no del artículo en su designación, el empleo del participio y de la oración de relativo, que son a veces el único medio de referencia al paciente, y, en fin, por otra parte, los diversos giros sintácticos, más o menos complejos, que se utilizan. Los resultados en todas estas cuestiones estudiadas apuntan en la misma dirección, y coinciden plenamente con los obtenidos en el examen de otros aspectos, de índole muy diversa; de modo que se corrobora una conexión mucho mayor entre los libros II y VI de Epidemias, frente a Epidemias IV, que se muestra en notable divergencia.

\section{BIBLIOGRAFÍA}

Alessi, Robert (2010), «Research Program and Teaching led by the Master in Hippocrates’ Epidemics II IV VI», en M. Horstmanshoff (ed.), Hippocrates and Medical Education. Selected papers presented at the XIIth Iinternational Hippocrates Colloquium, Leiden: 119-135.

ÁNGEL y EsPINÓs, Jesús (2002), «El proceso patológico en los tratados Epidemias V y VII según la expresión de la temporalidad», en A. Thivel, A. Zucker (edd.), Le normal et le pathologique dans la Collection hippocratique, $X^{e}$ Colloque International Hippocratique, Nice, t. II, 467-481.

- (2003): Comentario sintáctico-estilístico de Epidemias V y VII (tesis doctoral), Madrid, Servicio de Publicaciones de la Universidad Complutense de Madrid.

- (2009), «Algunas consideraciones acerca de ciertos rasgos sintácticos en Epidemias V y VII», Minerva 22: 107-126.

di Benedetto, Vincenzo (1977), «Principi metodici di Epidemie II.IV.VI», en R. Joly (ed.), Corpus Hippocraticum, Actes du Colloque Hippocratique de Mons, Mons: 246-263.

DeICHgräBer, Karl $\left(1971^{2}\right)$, Die Epidemien und das Corpus Hippocraticum, reimpr. BerlinNew York 1971 (Berlín 1933)

Esteban Santos, Alicia (1994), «Divergencias y paralelos entre las historias clínicas de Epidemias I y III», CFC (G) 4: 47-76.

- (1998), «Esquema composicional de las historias clínicas de Epid. V y VII. Encabezamiento: Quién, Dónde y Cuándo», en L. Gil, M Martínez Pastor, R. M. ${ }^{a}$ Aguilar (edd.) Corolla Complvtensis. Homenaje al Prof. José S. Lasso de la Vega, Madrid, 415-422.

- (2009), «Incisos dentro de las historias clínicas de Epidemias V y VII: los distintos tipos de pasajes», CFC (G) 19: 125-139.

- (2011), «Tipos de pasajes sobre enfermos individuales y su inserción en el todo en Epidemias II, IV y VI », CFC (G) 21: 179-192.

- (en prensa), «La denominación de los pacientes en Epidemias II, IV y VI», en L. M. Pino Campos y G. Santana Henríquez (edd.), Homenaje al Prof. Juan Antonio López Férez.

García Novo, Elsa (1989), «Introducción a los libros de Epidemias. Introducción a los libros II, IV y VI de Epidemias», en A. Esteban Santos, E. García Novo, B. Cabellos, Tratados hipocráticos V. Epidemias, Madrid: 7-19 y 131ss.

Grmek, Mirko D. (1980), «La description hippocratique de la "toux épidémique” de Périnthe», en M. D. Grmek (ed.), Hippocratica. Actes du Colloque hippocratique de Paris, Paris: 199-221. 
JouAnNA, Jacques (1989), «Place des Épidémies dans la Collection Hippocratique», en G. BAADER y R. WinAu (edd.), Die hippokratischen Epidemien, $V^{e}$ Colloque International Hippocratique, Stuttgart: pp. 60-87.

- (1992), Hippocrate, París.

LANGHOLF, Volker (1977): Syntaktische Untersuchungen zu Hippokrates-Texten. Brachylogische Syntagmen in den individuellen Krankeits-Fallbeschreibungen der hippokratischen Schriftensammlung. Wiesbaden.

- (1990), Medical theories in Hippocrates. Early texts and the «Epidemics». Berlin-New York.

LICCIARDI, Caterina (1989), «Tendence et probabilité dans les Épidémies II. IV. VI» en G. Baader, R. Winau (edd.), Die hippokratischen Epidemien, Ve Colloque International Hippocratique, Stuttgart: 117-130.

Manetti, Daniela \& Roselli, Amneris (1982), Epidemie. Libro sesto. Introduzione, testo critico, commento e traduzione. Firenze.

Nikitas, Anastasios A. (1968), Untersuchungen zu den Epidemienbüchern II IV VI des Corpus Hippocraticum (Tesis doctoral). Hamburg.

RoBert, Fernand (1975), «Les adresses de malades dans les Épidémies II, IV et VI», en La Collection Hippocratique et son rôle dans l'histoire de la médecine: colloque de Strasbour (Universite des sciences humaines de Strasbourg), Leiden: 173-194.

Sмiтн, Wesley D. (1989), "Generic form in Epidemics I to VII», en G. Baader, R. Winau (edd.), Die hippokratischen Epidemien, $V^{E}$ Colloque International Hippocratique, Stuttgart: 145-158.

— (1994), Hippocrates (Vol. VII). Epidemics 2, 4-7, Cambridge (Mass.)-London. 